\title{
Leaks of CP violation in the real two-Higgs-doublet model
}

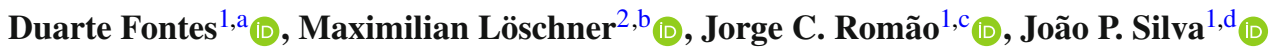 \\ ${ }^{1}$ Departamento de Física and Centro de Física Teórica de Partículas (CFTP), Instituto Superior Técnico (IST), U. de Lisboa (UL), \\ Av. Rovisco Pais, 1049-001 Lisbon, Portugal \\ ${ }^{2}$ Institute for Theoretical Physics, Karlsruhe Institute for Technology, Wolfgang-Gaede-Straße 1, 76131 Karlsruhe, Germany
}

Received: 15 March 2021 / Accepted: 13 June 2021 / Published online: 24 June 2021

(C) The Author(s) 2021

\begin{abstract}
We discuss the $Z_{2}$ symmetric two-Higgs-doublet model with a real soft-breaking term (real 2HDM). We explain in detail why it is not tenable to assume CP conservation in the scalar sector to keep the dimension two term real, while $\mathrm{CP}$ is violated by the dimension four Yukawa couplings. We propose the calculation of the infinite tadpole of the (would-be) pseudoscalar neutral scalar. We construct a simple toy model with the same flaws, where the unrenormalizable infinity is easier to calculate. We then consider the same tadpole in the real 2HDM. We spearhead this effort focusing on diagrams involving solely bare quantities. This involves hundreds of Feynman three-loop diagrams that could feed the CP violation from the quark into the scalar sector, and is only possible with state of the art automatic computation tools. Remarkably, some intermediate results agree when using three independent derivations, including the peculiar cancellation of the leading pole divergence due to a subtle interplay between masses and the Jarlskog invariant, which we calculate analytically. The calculation is not complete however, since the full two-loop renormalization of the real 2HDM is not yet available in the literature. Still, we argue convincingly that there is an irremovable infinity.
\end{abstract}

\section{Introduction}

The exact implementation of the symmetry breaking sector of the gauge theory describing fundamental particles is one of the most interesting open problems. In particular, the recent discovery at LHC of a $125 \mathrm{GeV}$ fundamental scalar $\left(h_{125}\right)$ $[1,2]$ begs the question of how many fundamental scalars there are in Nature. The Standard Model (SM) contains one single scalar doublet, but there is no fundamental requirement for this choice.

Indeed, there have been countless studies on models with two or more Higgs doublets; for reviews see [3-5] and references therein. The most studied model includes two Higgs doublets (2HDM) with a $Z_{2}$ symmetry, softly broken by a real parameter, with the additional requirement that the vacuum expectation values (vev) are taken as real. As such, the scalar sector by itself is $\mathrm{CP}$ conserving. The $Z_{2}$ symmetry is carried over to the fermions in such a way that only one scalar couples to all fermions of a given charge. The crucial point is that the experimentally observed CP violation [6] is accounted for by complex Yukawa couplings. This is sometimes referred to as the "real 2HDM", because the soft $Z_{2}$ breaking parameter is taken as real. Remarkably, this most prevalent model can suffer from theoretical inconsistencies regarding renormalization, a fact that is mostly ignored. ${ }^{1}$ This is the problem we address in detail here.

The paper is organized as follows. In Sect. 2, we discuss the inconsistency of requiring $\mathrm{CP}$ conservation in the potential of the real $2 \mathrm{HDM}$, while allowing for $\mathrm{CP}$ violation elsewhere. We argue that, at sufficient high order in perturbation theory, there could be divergences in CP-violating one-point and two-point functions that one can not remove by the counterterms provided by the theory. We show in Sect. 3 that this is precisely what happens in a toy model suffering from the same inconsistency as the real 2HDM. Then, in Sect. 4, we present the result for the leading pole of the three-loop onepoint function of the alleged CP-odd physical field of the real 2HDM. We describe in Sect. 5 the details of the different steps involved in the three-loop calculation. In Sect. 6, we summarize our conclusions.

\footnotetext{
a e-mail: duartefontes@ tecnico.ulisboa.pt (corresponding author)

b e-mail: maximilian.loeschner@kit.edu

c e-mail: jorge.romao@tecnico.ulisboa.pt

de-mail: jpsilva@cftp.ist.utl.pt
} 


\section{Shortcomings of the real $2 \mathrm{HDM}$}

Let us consider a $S U(2)_{L} \otimes U(1)_{Y}$ gauge theory with two Higgs-doublets $\Phi_{a}$, with the same hypercharge $1 / 2$, and with real vacuum expectation values (vevs)

$\left\langle\Phi_{a}\right\rangle=\left(\begin{array}{c}0 \\ v_{a} / \sqrt{2}\end{array}\right), \quad(a=1,2)$,

with $v=\sqrt{v_{1}^{2}+v_{2}^{2}}=246 \mathrm{GeV}$. Our definition for the charge is $Q=T_{3}+Y$, and we introduce the angle $\beta$ through

$\tan \beta=v_{2} / v_{1}$.

The most general 2HDM scalar potential may be written as

$$
\begin{aligned}
V_{H}= & m_{11}^{2} \Phi_{1}^{\dagger} \Phi_{1}+m_{22}^{2} \Phi_{2}^{\dagger} \Phi_{2}-\left[m_{12}^{2} \Phi_{1}^{\dagger} \Phi_{2}+\text { H.c. }\right] \\
& +\frac{1}{2} \lambda_{1}\left(\Phi_{1}^{\dagger} \Phi_{1}\right)^{2}+\frac{1}{2} \lambda_{2}\left(\Phi_{2}^{\dagger} \Phi_{2}\right)^{2}+\lambda_{3}\left(\Phi_{1}^{\dagger} \Phi_{1}\right)\left(\Phi_{2}^{\dagger} \Phi_{2}\right) \\
& +\lambda_{4}\left(\Phi_{1}^{\dagger} \Phi_{2}\right)\left(\Phi_{2}^{\dagger} \Phi_{1}\right)+\left[\frac{1}{2} \lambda_{5}\left(\Phi_{1}^{\dagger} \Phi_{2}\right)^{2}+\text { H.c. }\right] \\
& +\left[\lambda_{6}\left(\Phi_{1}^{\dagger} \Phi_{1}\right)\left(\Phi_{1}^{\dagger} \Phi_{2}\right)+\lambda_{7}\left(\Phi_{2}^{\dagger} \Phi_{2}\right)\left(\Phi_{1}^{\dagger} \Phi_{2}\right)+\text { H.c. }\right],
\end{aligned}
$$

where "H.c." stands for Hermitian conjugation. The coefficients $m_{11}^{2}, m_{22}^{2}$, and $\lambda_{1}, \ldots, \lambda_{4}$ are real while $m_{12}^{2}, \lambda_{5}, \lambda_{6}$ and $\lambda_{7}$ may be complex.

When extending this model to the fermion sector, one finds flavour changing neutral scalar interactions, which are very strongly constrained by experiments on neutral meson systems. This problem can be solved by imposing a $Z_{2}$ symmetry: $\Phi_{1} \rightarrow \Phi_{1} ; \Phi_{2} \rightarrow-\Phi_{2}[8,9]$. If the symmetry is exact, then the quadratic term $m_{12}^{2}=0$, and the quartic terms $\lambda_{6}=\lambda_{7}=0$. (The quartic term $\lambda_{5}$ can then be made real by a simple rephasing of $\Phi_{2}$.) This has the consequence that the model has no decoupling limit. That is, one cannot make arbitrarily large the masses of the new particles resultant from the presence of the second scalar doublet, thus approaching smoothly the SM limit. Such a decoupling is a desirable feature, especially since the couplings probed by current LHC data are consistent with the SM predictions, within errors of order 20\% [10]. Decoupling is recovered by reintroducing $m_{12}^{2} \neq 0$ [11], which, because it breaks softly the $Z_{2}$ symmetry, does not affect the renormalizability of the theory.

Most articles addressing this model then make the choice that $m_{12}^{2}$ and $\lambda_{5}$ are both real and the vevs are real, arguing that $\mathrm{CP}$ conservation in the scalar sector has been imposed (choice 1). Then one would proceed to discuss the various implementations of the $Z_{2}$ symmetry in the fermion sector, and perform a variety of fits to experiment. Among these, one must fit the well measured $\mathrm{CP}$ violation with origin in the Cabibbo-Kobayashi-Maskawa (CKM) matrix [12,13], accommodated by the complex Yukawa couplings (choice 2 ). The problem is that choice 1 and choice 2 are incompatible.

Indeed, either one applies the CP symmetry to the whole Lagrangian, in which case the Yukawa couplings are real and one cannot account for the observed $\mathrm{CP}$ violation; or, else, one does not apply CP symmetry anywhere, allowing the Yukawa couplings to be complex, but then allowing also for a complex $m_{12}^{2} \cdot{ }^{2}$ Said otherwise, requiring complex CKM and excluding the parameter $\operatorname{Im}\left(m_{12}^{2}\right)$ leads to a nonrenormalizable theory. At sufficiently high loop level, the $\mathrm{CP}$ violation in the quark sector will leak into the scalar sector, through a divergent contribution that cannot be absorbed by a $\operatorname{Im}\left(m_{12}^{2}\right)$ counterterm, which was absent from the theory from the start.

So why do all articles fitting the real 2HDM "model" to experiment ignore this problem? Because the divergent contribution can only be shown to happen in at least three loops. However, precisely because they are divergent, the problem cannot be ignored if one wishes to use a theoretically sound model.

Given the fact that the problem seems to occur due to (the lack of) $\operatorname{Im}\left(m_{12}^{2}\right)$, one could be tempted to assume that such a dimension two operator could not affect renormalizability. And indeed, it cannot affect renormalizability due to its soft-breaking of the $Z_{2}$ symmetry. But the problem with $\mathrm{CP}$ symmetry being invoked is not that it is broken by $m_{12}^{2}$ (real or complex); rather, it is (hard) broken by the dimension four Yukawa couplings.

It is true that one can look at the real $2 \mathrm{HDM}$ as a limiting case of the $Z_{2} 2 \mathrm{HDM}$, softly broken by a complex $m_{12}^{2}$. This model is known as the complex 2HDM (C2HDM) and has been studied in detail; see, for example [14-23]. In that case, one can choose any tree-level values for the parameters, and, in particular, set $\operatorname{Im}\left(m_{12}^{2}\right)=0$ at tree level. In that context, setting $\operatorname{Im}\left(m_{12}^{2}\right)=0$ at tree level, does not constitute a problem, since the theory does have its counterterm and is renormalizable. Is this the same as the real 2HDM? No: setting $\operatorname{Im}\left(m_{12}^{2}\right)=0$ in the C2HDM means that we are studying a very specific corner of tree-level parameter space of a more general model. The real $2 \mathrm{HDM}$, where there is no $\operatorname{Im}\left(m_{12}^{2}\right)$ nor its counterterm, is not a consistent model.

There is a more physical way to state the non renormalization problem. In any $2 \mathrm{HDM}$ there are three neutral scalars $\left(h_{1}, h_{2}\right.$, and $h_{3}$ ), and a pair of charged scalars $H^{ \pm}$. Typically, it is assumed that the lightest neutral scalar $\left(h_{1}\right)$ coincides with the $125 \mathrm{GeV}$ particle found at $\mathrm{LHC} .^{3}$ In the real $2 \mathrm{HDM}$, the (proclaimed) lack of $\mathrm{CP}$ violation in the scalar sector, leads to the separation of the three neutral scalars into one

\footnotetext{
$\overline{2}$ Recall that $\lambda_{5}$ can always be made real through a convenient rephasing of $\Phi_{2}$. In fact, one could instead use the rephasing to make $m_{12}^{2}$ real, at the price of getting a complex $\lambda_{5}$. What really matters is the rephasing invariant quantity $\operatorname{Im}\left[\lambda_{5}^{*}\left(m_{12}^{2}\right)^{2}\right]$. For simplicity, we will make the discussion in the basis where $\lambda_{5}$ is real.

3 This is not mandatory. One can accommodate the possibility that the $125 \mathrm{GeV}$ particle is not the lightest neutral scalar, but we shall not concern ourselves here with that case. See for example [24,25].
} 
single $\mathrm{CP}$ odd scalar $(A)$ and two $\mathrm{CP}$ even (usually denoted by $h$ for the lightest and $H$ for the heaviest). If the CKM $\mathrm{CP}$ violation indeed seeps into the scalar sector, then there should be divergent contributions to the $h-A$ and $H$ - $A$ twopoint functions. There will also be divergent contributions to the A tadpole. Since such terms are absent from the real scalar sector at tree level, there are no counterterms to absorb those infinities, and the theory is formally inconsistent.

\subsection{The scalar sector}

We start from the scalar sector of the real 2HDM

$$
\begin{aligned}
V_{r} & =m_{11}^{2} \Phi_{1}^{\dagger} \Phi_{1}+m_{22}^{2} \Phi_{2}^{\dagger} \Phi_{2}-m_{12}^{2}\left[\Phi_{1}^{\dagger} \Phi_{2}+\Phi_{2}^{\dagger} \Phi_{1}\right] \\
& +\frac{1}{2} \lambda_{1}\left(\Phi_{1}^{\dagger} \Phi_{1}\right)^{2}+\frac{1}{2} \lambda_{2}\left(\Phi_{2}^{\dagger} \Phi_{2}\right)^{2}+\lambda_{3}\left(\Phi_{1}^{\dagger} \Phi_{1}\right)\left(\Phi_{2}^{\dagger} \Phi_{2}\right) \\
& +\lambda_{4}\left(\Phi_{1}^{\dagger} \Phi_{2}\right)\left(\Phi_{2}^{\dagger} \Phi_{1}\right)+\frac{1}{2} \lambda_{5}\left[\left(\Phi_{1}^{\dagger} \Phi_{2}\right)^{2}+\left(\Phi_{2}^{\dagger} \Phi_{1}\right)^{2}\right],
\end{aligned}
$$

with all parameters real, and we parametrize the fields in the original basis as

$$
\begin{gathered}
\Phi_{1}=\left(\begin{array}{c}
c_{\beta} G^{+}-s_{\beta} H^{+} \\
\frac{1}{\sqrt{2}}\left[v c_{\beta}+\left(c_{\alpha} H-s_{\alpha} h\right)+i\left(c_{\beta} G^{0}-s_{\beta} A\right)\right]
\end{array}\right), \\
\Phi_{2}=\left(\begin{array}{c}
s_{\beta} G^{+}+c_{\beta} H^{+} \\
\frac{1}{\sqrt{2}}\left[v s_{\beta}+\left(s_{\alpha} H+c_{\alpha} h\right)+i\left(s_{\beta} G^{0}+c_{\beta} A\right)\right]
\end{array}\right) .
\end{gathered}
$$

Throughout $c_{\theta}=\cos \theta$ and $s_{\theta}=\sin \theta$, for whatever angle $\theta$ appears as a sub-index. Comparing Eq. (1) and Eq. (5), we recognize the choice $v_{1}=v c_{\beta}$ and $v_{2}=v s_{\beta}$. With that choice, $G^{+}$and $G^{0}$ will be massless and $H^{+}$is the physical charged scalar of mass $m_{H^{ \pm}}^{2}{ }^{4}$ These facts are confirmed by substituting Eq. (5) in Eq. (4). Performing the expansion, one sees that there is no linear term in $A$ (and, thus, no possibility to absorb any infinities that might appear in A tadpoles at loop level), nor are there any quadratic $h A$ or $H A$ terms (and, similarly, no possibility to absorb any infinities that might appear in the corresponding two-point functions at loop level).

The expansion does contain linear (tadpole) terms for $\mathrm{H}$ $\left(t_{H}\right)$ and $\mathrm{h}\left(t_{h}\right)$. Equating these tadpoles to zero, one obtains the same conditions that one would obtain by finding the stationarity conditions $\partial V_{r} / \partial v_{1}=0$ and $\partial V_{r} / \partial v_{2}=0$ directly from Eq. (4). Those two equations can be solved for $m_{11}^{2}$ and $m_{22}^{2}$, which are then substituted back into the expression for the potential. After this substitution using the vacuum conditions, there are no quadratic $G^{0} G^{0}$ and $G^{+} G^{-}$terms. There are also no mixed $G^{0} A$ or $G^{ \pm} H^{\mp}$ terms, meaning that as expected $G^{0}$ and $G^{+}$are Goldstone bosons, while $A$ and

\footnotetext{
4 This is in fact a feature of the general 2HDM (which can be extended to multiple doublets) related to the existence of a "Higgs basis" [26] $H_{1}=v_{1}^{*} \Phi_{1}+v_{2}^{*} \Phi_{2}, H_{2}=-v_{2} \Phi_{1}+v_{1} \Phi_{2}-$ where all the vev is in the first doublet [27,28].
}

$H^{+}$are already mass eigenstates. One finds quadratic terms of the type $H H, h h$, and $h H$. The angle $\alpha$ is chosen to kill the latter, meaning that $h$ and $H$ are the physical fields. Using $v=246 \mathrm{GeV}$ and $m_{h}=125 \mathrm{GeV}$, the scalar sector of the real $2 \mathrm{HDM}$ is parametrized by six further parameters: the mixing angles $\alpha$ and $\beta$; the masses $m_{H}, m_{A}, m_{H^{ \pm}}$; and the soft-breaking parameter $m_{12}^{2}=\operatorname{Re}\left(m_{12}^{2}\right)$.

\subsection{CP violation from the CKM matrix}

In the SM and in the real 2HDM, CP violation arises from the complex Yukawa couplings. When the quark fields are rotated into their mass basis, all $\mathrm{CP}$ violation phases are contained in the CKM matrix $[12,13]$. But, each quark field can still be rephased at will, thus moving the $\mathrm{CP}$ violating phase around the various entries of the CKM matrix. The only rephasing invariant quantity is [29-31]

$I_{\beta j}^{\alpha i}=\operatorname{Im}\left(V_{\alpha i} V_{\beta j} V_{\alpha j}^{*} V_{\beta i}^{*}\right)$,

where $\alpha \neq \beta$ and $i \neq j$. We use the notation of [32], where Greek letters $\alpha, \beta, \gamma, \ldots$ refer to up-type quarks $u_{\alpha}=u, c, t$, while Roman letters $i, j, k, \ldots$ refer to down-type quarks $d_{i}=d, s, b$. There are nine distinct four quark combinations with different flavours: $(d s),(d b)$, and $(s b)$ for the downtype quarks times the three for up-type quarks: $(u c),(u t)$, and $(c t)$. Using the unitarity of the CKM matrix, the following symmetries hold

$I_{\beta j}^{\alpha i}=I_{\alpha i}^{\beta j}=-I_{\beta i}^{\alpha j}=-I_{\alpha j}^{\beta i}$,

showing that indeed there is only one independent $\mathrm{CP}$ violating quantity. The antisymmetry with respect to interchange of same quark-type indices is easiest to see in the form

$I_{\beta j}^{\alpha i}=J \sum_{\gamma, k} \epsilon_{\alpha \beta \gamma} \epsilon_{i j k}$,

where $J$ is the Jarlskog invariant [29-31], defined for example as $J=I_{c s}^{u d}$. Notice that $I_{c s}^{u d} \neq 0$ even though only quarks from the first two families are involved. This does not contradict the fact that there would be no CP violating phase in the SM if there existed only two families of quarks. The fact that the CKM matrix is $3 \times 3$ unitary (and, thus, has one irremovable complex phase) is built into Eq. (8). Nevertheless, in $\mathrm{CP}$ violating processes with no external quarks (and, thus, a quark loop), the appearance of four CKM $(V)$ factors can only occur in diagrams at the three loop level and above.

As emphasized by Pospelov and Khriplovich [33] and by Booth [34] in the context of the electric dipole moments (edm) of the $W$ and the electron, the antisymmetry of $I_{\beta j}^{\alpha i}$ is very powerful. Any $\mathrm{CP}$ violating amplitude from a fermion box diagram will appear as the product $I_{\beta j}^{\alpha i}$ with some amplitude

$\mathcal{A}\left(m_{u_{\alpha}}, m_{u_{\beta}}, m_{d_{i}}, m_{d_{j}}\right)$. 
When all contributions are summed over $(\alpha, \beta, i$, and $j)$, all terms in $\mathcal{A}\left(m_{u_{\alpha}}, m_{u_{\beta}}, m_{d_{i}}, m_{d_{j}}\right)$ symmetric under $\alpha \leftrightarrow \beta$, or $i \leftrightarrow j$ will not contribute. A much more involved analysis along these lines was used in $[33,34]$ to show that the SM electroweak contributions to the electric dipole moments of the $W$ and the electron vanish to two-loop and three-loop approximation, respectively.

\section{A theoretically unsound toy model}

To better illustrate our claim, we consider here a toy model that suffers from the same inconsistency as the real 2HDM. In both models, CP conservation is enforced in a particular sector of an otherwise $\mathrm{CP}$ violating theory. As a consequence, $\mathrm{CP}$ violating radiative effects end up contaminating the alleged $\mathrm{CP}$ conserving sector, thus leading to divergences that cannot be absorbed by the counterterms. The major feature of our toy model is that such divergences show up immediately at one-loop order. Therefore, it constitutes a simple materialization of the same theoretical pathology that we claim to be present at three-loop or above in the real 2HDM.

The present model is inspired in a model by Pilaftsis [35], which, however, does not suffer from the flaw we wish to point out. Consider two Abelian gauge symmetries $U(1)_{Q}$ and $U(1)_{B}$, with gauge bosons $A_{\mu}$ and $B_{\mu}$, respectively. Suppose also four complex scalars, $\Phi_{1}, \Phi_{2}, \chi_{L}$ and $\chi_{R}$, with charges

$$
\begin{aligned}
& Q\left(\Phi_{1}\right)=0, Q\left(\Phi_{2}\right)=0, Q\left(\chi_{L}\right)=1, Q\left(\chi_{R}\right)=1, \\
& B\left(\Phi_{1}\right)=1, B\left(\Phi_{2}\right)=1, B\left(\chi_{L}\right)=-\frac{1}{5}, B\left(\chi_{R}\right)=\frac{4}{5},
\end{aligned}
$$

where $Q$ and $B$ represent the conserved charges of $U(1)_{Q}$ and $U(1)_{B}$, respectively. A discrete symmetry $D$ is imposed on the fields, under which:

$\Phi_{1} \stackrel{D}{\rightarrow}-\Phi_{1}, \quad \Phi_{2} \stackrel{D}{\rightarrow} \Phi_{2}, \quad \chi_{L} \stackrel{D}{\rightarrow}-\chi_{L}, \quad \chi_{R} \stackrel{D}{\rightarrow} \chi_{R}$.

However, $D$ is allowed to be softly broken. The complete renormalizable Lagrangian can then be written in four terms,

$\mathcal{L}=\mathcal{L}_{k i n}+\mathcal{L}_{\Phi}+\mathcal{L}_{\chi}+\mathcal{L}_{\Phi \chi}$,

where $\mathcal{L}_{\text {kin }}$ represents the kinetic terms ${ }^{5}$ and

$$
\begin{aligned}
-\mathcal{L}_{\Phi}= & \mu_{1}^{2} \Phi_{1}^{*} \Phi_{1}+\mu_{2}^{2} \Phi_{2}^{*} \Phi_{2}+\mu^{2} \Phi_{1}^{*} \Phi_{2}+\left(\mu^{2}\right)^{*} \Phi_{2}^{*} \Phi_{1} \\
& +\lambda_{1}\left(\Phi_{1}^{*} \Phi_{1}\right)^{2}+\lambda_{2}\left(\Phi_{2}^{*} \Phi_{2}\right)^{2}+\lambda_{34} \Phi_{1}^{*} \Phi_{1} \Phi_{2}^{*} \Phi_{2} \\
& +\lambda_{5}\left(\Phi_{1}^{*} \Phi_{2}\right)^{2}+\lambda_{5}^{*}\left(\Phi_{2}^{*} \Phi_{1}\right)^{2},
\end{aligned}
$$

$$
\begin{aligned}
-\mathcal{L}_{\chi}= & m_{L}^{2} \chi_{L} \chi_{L}^{*}+m_{R}^{2} \chi_{R} \chi_{R}^{*}+\rho_{1}\left(\chi_{L}^{*} \chi_{L}\right)^{2} \\
& +\rho_{2}\left(\chi_{R}^{*} \chi_{R}\right)^{2}+\rho_{34} \chi_{L}^{*} \chi_{L} \chi_{R}^{*} \chi_{R}, \\
-\mathcal{L}_{\Phi \chi}= & f_{1} \Phi_{1} \chi_{L} \chi_{R}^{*}+f_{1}^{*} \Phi_{1}^{*} \chi_{L}^{*} \chi_{R}+f_{2} \Phi_{2} \chi_{L} \chi_{R}^{*} \\
& +f_{2}^{*} \Phi_{2}^{*} \chi_{L}^{*} \chi_{R}+g_{1} \Phi_{1}^{*} \Phi_{1} \chi_{L}^{*} \chi_{L}+g_{2} \Phi_{2}^{*} \Phi_{2} \chi_{L}^{*} \chi_{L} \\
& +g_{3} \Phi_{1}^{*} \Phi_{1} \chi_{R}^{*} \chi_{R}+g_{4} \Phi_{2}^{*} \Phi_{2} \chi_{R}^{*} \chi_{R} .
\end{aligned}
$$

The parameters $\mu^{2}, \lambda_{5}, f_{1}$ and $f_{2}$ are in general complex, while the remaining ones are real by construction. The terms involving $\mu^{2}$ and $f_{2}$ break the symmetry $D$ softly. It is easy to show that the conditions for $\mathrm{CP}$ conservation are:

$$
\begin{aligned}
\operatorname{Im}\left[\mu^{2} f_{1} f_{2}^{*}\right] & =0, \\
\operatorname{Im}\left[\lambda_{5} f_{1}^{2}\left(f_{2}^{*}\right)^{2}\right] & =0, \\
\operatorname{Im}\left[\lambda_{5}^{*}\left(\mu^{2}\right)^{2}\right] & =0 .
\end{aligned}
$$

Mimicking the usual real 2HDM treatment, we take $\left\langle\Phi_{1}\right\rangle$ and $\left\langle\Phi_{2}\right\rangle$ real and parametrize

$\Phi_{1}=\frac{1}{\sqrt{2}}\left(v_{1}+H_{1}+i A_{1}\right)$,

$\Phi_{2}=\frac{1}{\sqrt{2}}\left(v_{2}+H_{2}+i A_{2}\right)$,

where $v_{1}, v_{2}$ are real and non-negative, and $H_{1}, H_{2}, A_{1}$, and $A_{2}$ are real fields. The vevs $v_{1}$ and $v_{2}$ break spontaneously the gauge symmetry $U(1)_{B}$. Recall that $\mu^{2}, \lambda_{5}, f_{1}, f_{2}$ are in general complex.

But suppose we force $\mathrm{CP}$ to be conserved in $\mathcal{L}_{\Phi}$ by the ad-hoc imposition that $\mu^{2}$ and $\lambda_{5}$ are real. This we will do in the following. It will lead to irremovable divergences at one-loop, as we now show.

We start by determining the minimization (or tadpole) equations. These are: ${ }^{6}$

$$
\begin{aligned}
0 & =t_{H_{1}}:=\left.\frac{\partial \mathcal{L}_{\Phi}}{\partial H_{1}}\right|_{<>=0} \\
& =-v_{1}\left(\mu_{1}^{2}+\frac{v_{2}}{v_{1}} \mu^{2}+v_{1}^{2} \lambda_{1}+\frac{1}{2} v_{2}^{2} \lambda_{34}+v_{2}^{2} \lambda_{5}\right), \\
0 & =t_{H_{2}}:=\left.\frac{\partial \mathcal{L}_{\Phi}}{\partial H_{2}}\right|_{<>=0} \\
& =-v_{2}\left(\mu_{2}^{2}+\frac{v_{1}}{v_{2}} \mu^{2}+v_{2}^{2} \lambda_{2}+\frac{1}{2} v_{1}^{2} \lambda_{34}+v_{1}^{2} \lambda_{5}\right),
\end{aligned}
$$

where $t_{H_{1}}, t_{H_{2}}$ represent the tree-level tadpoles for $H_{1}, H_{2}$, respectively, and $<>=0$ means that the expectation values of all fields on the right hand side of Eqs. (16) are set to zero. Recall that we are taking $\mu^{2}$ and $\lambda_{5}$ as real parameters. For that reason, the mass matrices for $H_{1}$ and $H_{2}$, on the one hand, and $A_{1}$ and $A_{2}$, on the other, can be separately

\footnotetext{
${ }^{6}$ The tadpole equations for $A_{1}$ and $A_{2}$ are trivially zero.
} 
diagonalized. We thus define the angles $\theta$ and $\beta$ such that:

$$
\begin{aligned}
\left(\begin{array}{l}
H_{1} \\
H_{2}
\end{array}\right) & =\left(\begin{array}{cc}
c_{\theta} & -s_{\theta} \\
s_{\theta} & c_{\theta}
\end{array}\right)\left(\begin{array}{l}
h \\
H
\end{array}\right), \\
\left(\begin{array}{l}
A_{1} \\
A_{2}
\end{array}\right) & =\left(\begin{array}{cc}
c_{\beta} & -s_{\beta} \\
s_{\beta} & c_{\beta}
\end{array}\right)\left(\begin{array}{c}
G^{0} \\
A
\end{array}\right),
\end{aligned}
$$

where $h$ and $H$ are the $\mathrm{CP}$ even states, and $A$ and $G^{0}$ are the CP odd states, where $G^{0}$ is the massless would-be Goldstone boson. ${ }^{7}$ We continue using the short notation $s_{x} \equiv \sin (x)$, $c_{x} \equiv \cos (x)$, for a generic angle $x$. As for the $\mathcal{L}_{\chi}$ sector, the mass matrix is given by

$$
-\mathcal{L}_{\text {mass }}^{\chi}=\left(\chi_{L}^{*} \chi_{R}^{*}\right)\left(\begin{array}{ll}
a & b^{*} \\
b & c
\end{array}\right)\left(\begin{array}{l}
\chi_{L} \\
\chi_{R}
\end{array}\right)
$$

with

$a=\frac{1}{2} g_{1} v_{1}^{2}+\frac{1}{2} g_{2} v_{2}^{2}+m_{L}^{2}$,

$b=\frac{f_{1} v_{1}+f_{2} v_{2}}{\sqrt{2}}$,

$c=\frac{1}{2} g_{3} v_{1}^{2}+\frac{1}{2} g_{4} v_{2}^{2}+m_{R}^{2}$,

where $a$ and $c$ are real, while $b$ is in principle complex. However, we can rephase $\chi_{R}$ through $\chi_{R} \rightarrow e^{i \arg (b)} \chi_{R}$ so that it absorbs the phase of $b$. In the new basis, then, $b$ is real, which implies that the mass matrix in Eq. (19) is symmetric. ${ }^{8}$ We thus need an orthogonal matrix with a new angle $\phi$ to diagonalize the states:

$$
\left(\begin{array}{l}
\chi_{L} \\
\chi_{R}
\end{array}\right)=\left(\begin{array}{cc}
c_{\phi} & -s_{\phi} \\
s_{\phi} & c_{\phi}
\end{array}\right)\left(\begin{array}{l}
\chi_{1} \\
\chi_{2}
\end{array}\right)
$$

where $\chi_{1}$ and $\chi_{2}$ are the (complex) diagonalized states with (real) masses $M_{1}$ and $M_{2}$.

When considering the theory up to one-loop level, one should renormalize it in order to obtain finite $S$-matrix elements. This is done through the usual procedure: taking an independent set of parameters, identifying them with bare quantities (represented in the following with the index 0) and relating them to their renormalized equivalents through a counterterm. Tadpoles can be taken care of through the tadpole scheme identified by PRTS in [36]. It then follows from the set of Eqs. (17) that:

\footnotetext{
${ }_{7} G^{0}$ is eaten by the longitudinal component of $B_{\mu}$ in the unitary gauge through the Higgs mechanism. The fact that $G^{0}$ is massless forces $\beta$ to obey the relation $\tan \beta=\frac{v_{2}}{v_{1}}$.

${ }^{8}$ If it were hermitian, one would need a unitary matrix to diagonalize it, instead of an orthogonal one. Moreover, note that $b$ real forces the relation $f_{1}^{*}=f_{1}+\left(f_{2}-f_{2}^{*}\right) \tan \beta$.
}
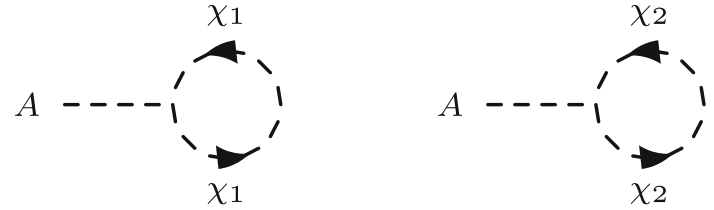

Fig. 1 Feynman diagrams contributing to the one-loop tadpole of the $\mathrm{CP}$ odd state

$\delta t_{H_{1}}:=-v_{1}\left(\mu_{1,0}^{2}+\frac{v_{2}}{v_{1}} \mu_{0}^{2}+v_{1}^{2} \lambda_{1,0}+\frac{1}{2} v_{2}^{2} \lambda_{34,0}+v_{2}^{2} \lambda_{5,0}\right)$,

$\delta t_{H_{2}}:=-v_{2}\left(\mu_{2,0}^{2}+\frac{v_{1}}{v_{2}} \mu_{0}^{2}+v_{2}^{2} \lambda_{2,0}+\frac{1}{2} v_{1}^{2} \lambda_{34,0}+v_{1}^{2} \lambda_{5,0}\right)$,

where the tadpole counterterms $\delta t_{H_{1}}$ and $\delta t_{H_{2}}$ are such that $\delta t_{H_{1}}=-T_{H_{1}}$ and $\delta t_{H_{2}}=-T_{H_{2}}$, with $T_{H_{1}}$ and $T_{H_{2}}$ being the one-loop tadpole for $H_{1}$ and $H_{2}$, respectively. The set of (22) fixes the values for $v_{1}$ and $v_{2}$ at one-loop level. Note that, since we imposed CP conservation in $\mathcal{L}_{\Phi}$, there are no tadpole counterterms for the CP odd fields. Specifically, in the mass basis,

$\delta t_{A}=0$.

But it is easy to see that this is inconsistent. Indeed, there is a one-loop tadpole for $A$, whose diagrams are represented in Fig. 1. The sum of diagrams is divergent. In fact,

$\left.\left(T_{A}\right)\right|_{\mathrm{div}}=-\frac{1}{\varepsilon} \frac{c_{\phi} s_{\phi}\left(M_{1}^{2}-M_{2}^{2}\right) \operatorname{Im}\left[f_{2}\right]}{8 \sqrt{2} \pi^{2} c_{\beta}}$,

in $d=4-2 \varepsilon$ dimensions, where $T_{A}$ represents the one-loop tadpole for $A$ and $\left.\right|_{\text {div }}$ means that we consider only divergent parts.

The origin of the problem lies in the fact that we imposed $\mu^{2}$ and $\lambda_{5}$ to be real. To clarify this point, let us provisionally take these parameters to be complex, as they originally were. By rewriting Eq. (14a) in terms of bare quantities, and separating the real and imaginary parts of $\mu_{0}^{2}$ and $\lambda_{5,0}$, the terms proportional to these parameters are:

$$
\begin{aligned}
- & \mathcal{L}_{\Phi_{0}} \ni \mu_{0}^{2} \Phi_{1,0}^{*} \Phi_{2,0}+\left(\mu_{0}^{2}\right)^{*} \Phi_{2,0}^{*} \Phi_{1,0} \\
& +\lambda_{5,0}\left(\Phi_{1,0}^{*} \Phi_{2,0}\right)^{2}+\lambda_{5,0}^{*}\left(\Phi_{2,0}^{*} \Phi_{1,0}\right)^{2} \\
= & \operatorname{Re}\left[\mu_{0}^{2}\right]\left(\Phi_{1,0}^{*} \Phi_{2,0}+\Phi_{2,0}^{*} \Phi_{1,0}\right) \\
& +\operatorname{Re}\left[\lambda_{5,0}\right]\left\{\left(\Phi_{1,0}^{*} \Phi_{2,0}\right)^{2}+\left(\Phi_{2,0}^{*} \Phi_{1,0}\right)^{2}\right\} \\
& +i \operatorname{Im}\left[\mu_{0}^{2}\right]\left(\Phi_{1,0}^{*} \Phi_{2,0}-\Phi_{2,0}^{*} \Phi_{1,0}\right) \\
& +i \operatorname{Im}\left[\lambda_{5,0}\right]\left\{\left(\Phi_{1,0}^{*} \Phi_{2,0}\right)^{2}-\left(\Phi_{2,0}^{*} \Phi_{1,0}\right)^{2}\right\} .
\end{aligned}
$$

As a consequence, when we set $\operatorname{Im}\left[\mu_{0}^{2}\right]=\operatorname{Im}\left[\lambda_{5,0}\right]=0$, we are not including in the model the terms of the two last 


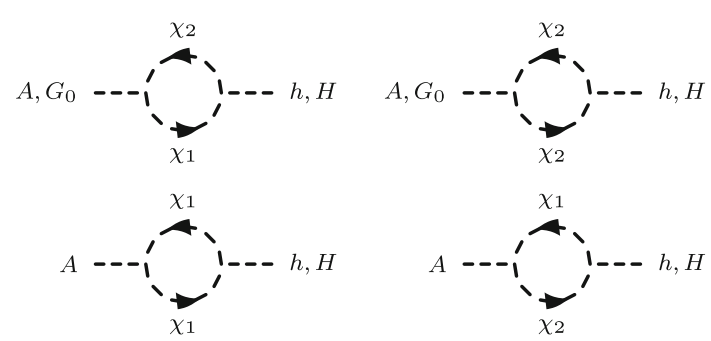

Fig. 2 Feynman diagrams contributing to the one-loop CP violating 2-point functions

lines of Eq. (25). Naturally, since such terms are not in the model, there is no counterterm for the parameters involved therein. That is, there is neither $\operatorname{Im}\left[\delta \mu^{2}\right]$ nor $\operatorname{Im}\left[\delta \lambda_{5}\right] .{ }^{9}$ Now, it is a matter of course that this would not be a problem if the fact that we did not include the terms in the last two lines of Eq. (25) would follow from a symmetry that forbade them. In other words, should there be a symmetry in the theory that proscribed those terms, they could logically not be included; and since the symmetry would prevent any Green's functions generated by such terms from showing up, there would never be divergences involved therein, so that the absence of counterterms for them would never be a problem. So, for example, if $\mathrm{CP}$ was a symmetry of the theory, it would preclude those terms, in which case the absence of $\operatorname{Im}\left[\delta \mu^{2}\right]$ and $\operatorname{Im}\left[\delta \lambda_{5}\right]$ would not be inconsistent.

However, CP is not a symmetry of theory: even if we try to impose it in the $\mathcal{L}_{\Phi}$ sector, it still is violated in the $\mathcal{L}_{\Phi \chi}$ sector through the phases of $f_{1}$ and $f_{2}$, as Eqs. (15a) and (15b) show. So, there is no CP symmetry forbidding the terms in the last two lines of Eq. (25). As a consequence, even if we exclude them, $\mathrm{CP}$ violating radiative effects can nonetheless contribute to the Green's functions involved therein. Such Green's functions will in general be divergent; but since the last two lines of Eq. (25) were not included in the theory, there will in general not be enough counterterms to absorb them.

We have already seen one example of Green's function that indeed cannot be renormalized: the one-loop 1-point function $T_{A}$. Other examples are the one-loop CP violating 2-point functions $\Sigma^{G^{0} h}, \Sigma^{G^{0} H}, \Sigma^{A h}$ and $\Sigma^{A H}$ for the scalarpseudoscalar mixing of $G^{0} h, G^{0} H, A h$ and $A H$, respectively. Their Feynman diagrams are represented in Fig. 2.

Just like in the case of $T_{A}$, there simply is no counterterm for these functions, which nonetheless are divergent. Their

\footnotetext{
9 The situation would not be different if we decided to exclude any other term from the theory. For example, had we decided not to include the term proportional to $\lambda_{1}$ in the model, there would be no counterterm $\delta \lambda_{1}$.
}

divergent parts are:

$$
\begin{aligned}
& \left.\Sigma^{G^{0} h}\left(k^{2}\right)\right|_{\mathrm{div}}=\left.\Sigma^{A H}\left(k^{2}\right)\right|_{\mathrm{div}} \\
& =-\frac{1}{\varepsilon} \sin (\beta-\theta) \frac{\operatorname{Im}\left[f_{2}\right]\left(f_{1}+f_{2} \tan \beta\right)}{16 \pi^{2}}, \\
& \left.\Sigma^{A h}\left(k^{2}\right)\right|_{\mathrm{div}}=-\left.\Sigma^{G^{0} H}\left(k^{2}\right)\right|_{\mathrm{div}} \\
& =-\frac{1}{\varepsilon} \cos (\beta-\theta) \frac{\operatorname{Im}\left[f_{2}\right]\left(f_{1}+f_{2} \tan \beta\right)}{16 \pi^{2}} .
\end{aligned}
$$

In conclusion, the fact that we imposed $\mu^{2}$ and $\lambda_{5}$ to be real leads to several divergences that cannot be removed by counterterms.

There are two ways to heal this model: either $\mathrm{CP}$ is imposed as a whole, or it is not imposed at all. In the first case, all the three relations in Eqs. (15) should be verified, which implies that there is a basis where $\mu^{2}, \lambda_{5}, f_{1}$ and $f_{2}$ are all real. In this scenario, therefore, CP violating Green's functions are precluded, which implies, in particular, that no divergent $\mathrm{CP}$ violating Green's functions will appear in any order. This is consistent with what we obtained in Eqs. (24) and (26), which vanish in the limit of real $f_{1}$ and $f_{2}$. In the second case, $\mu^{2}, \lambda_{5}, f_{1}$ and $f_{2}$ are in general complex parameters, which implies that their counterterms are also in general complex. Since CP is violated, there are no scalar states with well-defined CP, and Green's functions will in general be CP violating. The model is renormalizable as long as all the terms compatible with the symmetries are included. Finally, note that, in such a $\mathrm{CP}$ violating scenario, there may be regions of the parameter space in which $\lambda_{5}$ and $\mu^{2}$ are real, and $f_{1}$ and $f_{2}$ complex. But this is a completely different situation from that where one builds a theory taking ab initio $\lambda_{5}$ and $\mu^{2}$ real, while $f_{1}$ and $f_{2}$ in general complex. In fact, while the former situation corresponds to a particular solution of a consistent, renormalizable theory, the latter suffers from the inconsistencies we have shown.

\section{Three-loop tadpole for $A$ in the real 2HDM}

Our goal is to check whether the complex phases of the fermion mixing matrices introduce $\mathrm{CP}$ violating effects into the otherwise CP conserving scalar sector of the real 2HDM via radiative corrections. For this purpose, we focus on the effects of quark-mixing. The quantity that signifies quarkinduced CP violation in a convention independent way is the Jarlskog invariant $J$ in Eq. (8). So, we are looking for radiative corrections to the $2 \mathrm{HDM}$ which contain this quantity. As the simplest check, we have looked for diagrams proportional to $J$, contributing to the $A$ tadpole. As argued above, this can only happen in amplitudes with at least four vertices, each containing a factor of $V_{u_{\alpha}} d_{j}$ and additionally, a vertex to couple to $A$ (none of the $A$-couplings have CKM-factors). 


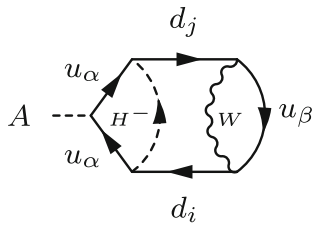

(a) (b)

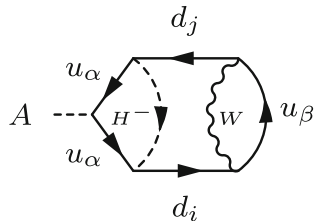

Fig. 3 Example of pair of Feynman diagrams where $J$ factorizes (for fixed $\alpha, \beta, i, j$ ). They differ only by the direction of fermion flow (or equivalently by the exchange of the two down-type quarks, $d_{i} \leftrightarrow d_{j}$ )

Therefore, the first possible appearance of $J$ in $A$-tadpoles is at three loops. This is indeed what we find at the amplitudelevel.

An example of a pair of diagrams yielding the Jarlskog invariant is shown in Fig. 3. If contributions of this kind were divergent, then one would lack the respective counterterm needed to absorb these divergences within the real 2HDM. Therefore, we checked for the existence of a leading $1 / \varepsilon^{3}$ pole in said contributions, which would be a strong indication for the necessity of a genuine three-loop $A$-tadpole counterterm. For this purpose, we generated all three-loop $A$-tadpole amplitudes $T_{A}$ with the only condition being that a fixed set of quarks $\left\{u_{\alpha}, u_{\beta}, d_{i}, d_{j}\right\}$ must be contained. Other contributions are $\mathrm{CP}$ conserving operators and, thus, irrelevant to our discussion.

Our calculation was carried out in three independent ways (two numeric; one analytical), fully explained in Sect. 5. The result is ${ }^{10}$

$$
\begin{aligned}
\left(T_{A}\right)_{\beta j}^{\alpha i} & \left.=-i(-)^{-}\right)_{u_{\beta} d_{j}}^{u_{\alpha} d_{i}} \\
& =\frac{g^{5}}{8 \varepsilon^{3} m_{W}^{3} s_{\beta} c_{\beta}} M_{\beta j}^{\alpha i} I_{\beta j}^{\alpha i}+\mathcal{O}\left(\varepsilon^{-2}\right),
\end{aligned}
$$

where there is no sum over repeated indices, and

$$
\begin{aligned}
M_{\beta j}^{\alpha i}= & \left(m_{u_{\alpha}}^{2}-m_{u_{\beta}}^{2}\right)\left(m_{d_{i}}^{2}-m_{d_{j}}^{2}\right) \\
& \times\left(m_{u_{\alpha}}^{2}-m_{d_{i}}^{2}+m_{u_{\beta}}^{2}-m_{d_{j}}^{2}\right) .
\end{aligned}
$$

The fact that such different calculational techniques yielded the same result is truly significant.

Remarkably, when summing over all different sets of upand down-type quark contributions, the leading pole vanishes exactly. Indeed, it is easy to show that summing the combination $M_{\beta j}^{\alpha i} I_{\beta j}^{\alpha i}$ over all the nine distinct sets of four

\footnotetext{
${ }^{10}$ Notice that the angle $\beta$ in $s_{\beta} c_{\beta}$ is the angle in Eq. (2), while in all other instances of Eq. (27), $\beta$ refers to the up-type quark being considered. Here and henceforth, which $\beta$ is meant should be clear from the context.
}

different quarks (two up-type and two down-type), the result vanishes. Notice that both $M_{\beta j}^{\alpha i}$ and $I_{\beta j}^{\alpha i}$ are antisymmetric under $\alpha \leftrightarrow \beta$ (or $i \leftrightarrow j$ ). Thus, the vanishing of Eq. (27) is not due to the simple symmetry reasons mentioned in connection with Eq. (9). It is the specific form of the mass term $M_{\beta j}^{\alpha i}$ in Eq. (28) which makes this possible. We cannot see how one would have guessed from the start this rather peculiar mass combination. We resonate with Khriplovich and Pospelov's remark in the context of edm that: "We cannot get rid of the feeling that this simple result (...) should have a simple transparent explanation. Unfortunately, we have not been able to find it."

But the physical consequence is quite clear:

$\sum_{\alpha<\beta} \sum_{i<j}\left(T_{A}\right)_{\beta j}^{\alpha i}=\mathcal{O}\left(\varepsilon^{-2}\right)$.

It remains uncertain whether this cancellation has a physical origin or it is to be interpreted as accidental. There is the possibility that the next order $1 / \varepsilon^{2}$-poles would be nonvanishing. Otherwise, a genuine $\mathrm{CP}$ violating tadpole counterterm for $A$ would only become relevant at the four-loop level.

\section{Details of the calculation}

In this section, we discuss our derivation of (27). First note that a complete calculation of the renormalized three-loop tadpole for $A$ would require the full renormalization of the model at both one-loop and two-loop order. Although unlikely, one cannot exclude the possibility that combinations of the one- and two-loop counterterms of a CPconserving scalar sector conspire to cancel the divergences of a CP-violating three-loop tadpole. Secondly, one caveat in our calculation is the treatment of amplitudes with an uneven number of $\gamma$-matrices together with $\gamma_{5}$. We chose to work in naive dimensional regularisation with the expectation that the leading $\varepsilon$-poles do not depend on the choice of a $\gamma_{5}$ scheme. This claim is supported by the findings in [37] at the one-loop level.

As mentioned before, at least three generations of quarks are necessary to generate a CP-violating tadpole. Therefore, we focus on a particular set of diagrams. Let us then define $S^{\{d c b t\}}$ as the set of all the three-loop tadpole diagrams for $A$ containing the quarks $d, c, b, t$. We started by generating the amplitudes for $S^{\{d c b t\}}$ in an $R_{\xi}$-gauge. We did this through two independent softwares: FeynMaster [38] (which makes use of FeynRules [39] and QGRAF [40]) and FeynArts [41]. ${ }^{11}$ At three loops, there are 360 amplitudes containing the quarks $d, c, b$ and $t$. However, 120 among

\footnotetext{
${ }^{11}$ It is worth emphasizing that the three-loop tadpole amplitudes generated with FeynArts and FeynMaster coincide.
} 


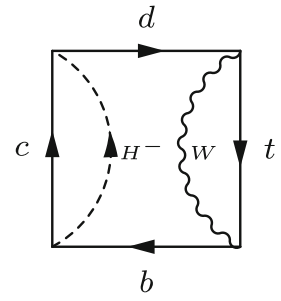

(a)

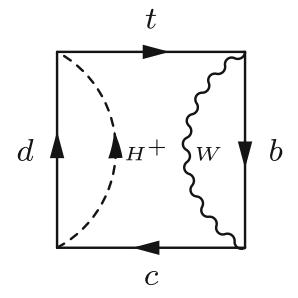

(b)
Fig. 4 Attaching $A$ to the fermion lines of these diagrams and the ones with reversed fermion flow generates the first category of relevant diagrams

them involve two closed loops of fermions, which means that they can never factorize the Jarlskog invariant $J$; and since a) the tadpole for $A$ violates $C P$ and b) all the $C P$ violation in the real 2HDM must be proportional to $J$ (with $J$ being the only rephasing-invariant quantity signifying CPviolation), those diagrams must sum up to zero. We checked this explicitly using FeynCalc [42-44].

We then focused on the remaining 240 diagrams. After simplifying the Lorentz and Dirac algebra of the 240 diagrams with FeynCalc, another 32 diagrams, such as the ones with two internal W-boson loops, vanish immediately (in naive dimensional regularisation) due to the chirality of the interactions involved. This eventually left us with 208 diagrams which can be categorized as follows:

1. The first group of diagrams can be generated from connecting $A$ to any fermion line in Fig. 4 and the corresponding ones with reversed fermion flow giving 16 diagrams. The same goes for all possible vector boson and scalar insertions, namely

\section{$\{H W, W H, H G, G H, H H, G W, W G, G G\}$.}

Diagrams where we connect $A$ to a line with an attached $W$-loop vanish though, leaving us with $8 \times 16-4 \times 4=$ 112 diagrams.

2. An example of the second group is shown in Fig. 5. From this diagram and the one with reversed fermion flow, we get 8 diagrams by cyclic permutations of the fermions. The $A$ can be connected to either $\{W H, H W, G H, H G\}$ and we can have either a $W$-, $H$-, or $G$-loop in the diagram. This gives $3 \times 4 \times 8=96$ diagrams.

We proceeded to numerically evaluate the most divergent part of the 208 diagrams using FIESTA [45] in Feynman gauge, i.e. at $\xi_{W}=1$. In order to generate input integrals, the FeynCalc function ApartFF was essential for decomposing the diagrams via partial fractioning. This decomposition yielded scalar integrals for which we could easily get an accurate result from FIESTA. One integral type needed addi-

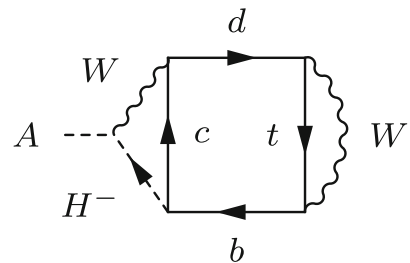

Fig. 5 The second set of relevant diagrams is generated from permutations of the fermions in this diagram and by replacing the $W$-insertions with a Goldstone boson

tional attention though, namely the one with a scalar product in the numerator and five different propagator factors (see Appendix A for a discussion). This type of integral yielded large error estimates in FIESTA, such that the results could no be trusted. In order to obtain an exact result for those, we used integration-by-parts identities with FIRE [46] to decompose this integral type into a set of scalar integrals. The intermediate steps required to link FeynCalc, FIRE and FIESTA were performed by two independent sets of private codes.

For the numerical input values of the scalar sector, one should choose a point in parameter space which does not violate any theoretical or experimental constraints. The theoretical bounds include boundedness from below, perturbative unitarity [47-49] as well as electroweak precision measurements using the oblique parameters S, T and U [4]. The experimental constraints include the exclusion bounds from Higgs searches at LHC that were verified using HiggsBounds $[50,51]$ and the signal strengths for the SM-like Higgs boson were forced to be within $2 \sigma$ of the fits given in $[10,52]$. Among the points that pass all constraints, we pick the following one:

$$
\begin{aligned}
\alpha & =-0.83797 \\
\beta & =0.73908 \\
m_{H^{ \pm}} & =581.18 \mathrm{GeV}, \\
m_{H} & =592.81 \mathrm{GeV}, \\
m_{A} & =597.44 \mathrm{GeV}, \\
m_{12}^{2} & =19.458 \mathrm{TeV} .
\end{aligned}
$$

Using these together with Eq. (B.1), the result for the most divergent part of $S^{\{d c b t\}}$ is:

$$
\left(i T_{A}\right)_{t b}^{c d}=2392.6(\mathrm{GeV})^{3} \times \frac{1}{\varepsilon^{3}} J+\mathcal{O}\left(\varepsilon^{-2}\right) .
$$

We tried to ascertain whether sets of diagrams with different combinations of quarks could possibly cancel with each other by checking the result for another set of quarks. For example, consider the set of diagrams $S^{\{d c s t\}}$, defined as identical to $S^{\{d c b t\}}$ except that the $b$ quark is replaced by an $s$ quark. Using the same point in parameter space (Eq. 30), the result 
for the most divergent part of $S^{\{d c s t\}}$ is

$$
\left(i T_{A}\right)_{t s}^{c d}=-0.91341(\mathrm{GeV})^{3} \times \frac{1}{\varepsilon^{3}} J+\mathcal{O}\left(\varepsilon^{-2}\right) .
$$

Clearly, the numbers differ, which might lead one to believe that summing over the results for all quarks combinations would likely yield a non-zero result.

Only later, our attention was drawn to TVID [53,54], a software package for the numerical evaluation of arbitrary three-loop vacuum integrals - see also [55]. The authors discuss a set of three master integrals into which any three-loop tadpole diagram can be decomposed. Using the same amplitude decomposition as for our numerical evaluation, we were then able to map to this set of master-integrals and use the analytic pole expressions of TVID (or equivalently of Ref. [56]) to acquire the result for the $1 / \varepsilon^{3}$-pole shown in (27). ${ }^{12}$ After confirming that the analytic results coincided with our previous numerical findings, we were able to sum over all possible quark combinations using (27) to find the surprising result of the poles cancelling, as shown in Eq. (29).

\section{Conclusions}

We argued that the real $2 \mathrm{HDM}$ can suffer from theoretical inconsistencies, as the simultaneous enforcement of $\mathrm{CP}$ conservation in the potential and allowance of $\mathrm{CP}$ violation in another sector may lead to divergences that cannot be removed by the counterterms. Because such divergences cannot show up at two-loop level and below, the unsoundness of the model has been by and large ignored in the literature. But the problem cannot be dismissed. In order to highlight its potential theoretical unsoundness we introduced a simple toy model, characterized by the same inconsistency as the real 2HDM. There, and as we showed, the irremovable divergences (that are expected at least at three-loops in the real 2HDM) show up immediately at one-loop level. This simple example ought to make the point: the real $2 \mathrm{HDM}$ could suffer from the same kind of pathology. We addressed this claim by calculating the leading pole of the three-loop one-point function of the $A$ field in the real $2 \mathrm{HDM}$. We showed that, surprisingly, the pole vanishes exactly after summing all contributions. This does not mean that the model is sound after all, but only that its unsoundness is likely to be found either at lower order in $1 / \varepsilon$ or upon two-loop renormalization, or possibly at four-loop order. A complete discussion would require the full one- and two-loop renormalization as well as a discussion of the $\gamma_{5}$-scheme beyond naive dimensional regularisation though.

\footnotetext{
12 This calculation also showed that the evaluation of scalar threeloop integrals with up to five propagators and different mass scales via FIESTA yields accurate results for the leading poles. This might be the first such stress test on this package.
}

We hope that our work spurs further interest in this subject and that a full calculation will become possible in the future.

Acknowledgements We are grateful to Francisco Botella, Renato Fonseca, Paulo Nogueira and Howard Haber for discussions, as well as to Vladyslav Shtabovenko and Alexander Smirnov for useful suggestions concerning softwares for multi-loop evaluation. This work is supported in part by the Portuguese Fundação para a Ciência e Tecnologia (FCT) under contracts UIDB/00777/2020, UIDP/00777/2020, CERN/FIS-PAR/0004/2017, and PTDC/FIS-PAR/29436/2017. D.F. is also supported by the Portuguese Fundação para a Ciência e Tecnologia under the project SFRH/BD/135698/2018. M.L. is supported partially by the DFG Collaborative Research Center TRR 257 "Particle Physics Phenomenology after the Higgs Discovery".

Data Availability Statement This manuscript has associated data in a data repository. [Authors' comment: All data in this manuscript are available upon request by contacting the corresponding author.]

Open Access This article is licensed under a Creative Commons Attribution 4.0 International License, which permits use, sharing, adaptation, distribution and reproduction in any medium or format, as long as you give appropriate credit to the original author(s) and the source, provide a link to the Creative Commons licence, and indicate if changes were made. The images or other third party material in this article are included in the article's Creative Commons licence, unless indicated otherwise in a credit line to the material. If material is not included in the article's Creative Commons licence and your intended use is not permitted by statutory regulation or exceeds the permitted use, you will need to obtain permission directly from the copyright holder. To view a copy of this licence, visit http://creativecomm ons.org/licenses/by/4.0/.

Funded by $\mathrm{SCOAP}^{3}$.

\section{Appendix A: Integral decomposition}

As mentioned in Sect. 5, one integral type in our amplitude decomposition needed special attention. The one in question is

$$
\begin{aligned}
& U_{5}^{(1,2)}\left(m_{1}, m_{2}, m_{3}, m_{4}, m_{5}\right) \\
& =i \frac{e^{3 \gamma_{E} \varepsilon}}{\pi^{3 d / 2}} \int \mathrm{d}^{d} q_{1} \mathrm{~d}^{d} q_{2} \mathrm{~d}^{d} q_{3} \frac{q_{1} \cdot q_{2}}{\left(q_{1}^{2}-m_{1}^{2}\right)\left(q_{2}^{2}-m_{2}^{2}\right)\left(q_{3}^{2}-m_{3}^{2}\right)} \\
& \quad \times \frac{1}{\left(\left(q_{1}-q_{3}\right)^{2}-m_{4}^{2}\right)\left(\left(q_{2}-q_{3}\right)^{2}-m_{5}^{2}\right)}
\end{aligned}
$$

Evaluating integrals of this kind with FIESTA yields small yet non-zero error estimates already for the leading pole which is why the numerical results could not be trusted. Therefore, we made use of the integration by parts routines of FIRE to decompose $U_{5}^{(1,2)}$ into a set of scalar integrals, which in turn yield vanishing error estimates for the leading poles when evaluated with FIESTA. Using analytic expressions for the leading poles of the resulting integral decomposition also made it possible to recombine everything into a joint expression for the leading pole of $U_{5}^{(1,2)}$, viz. 


$$
\begin{aligned}
& U_{5}^{(1,2)}\left(m_{1}, m_{2}, m_{3}, m_{4}, m_{5}\right) \\
& =\frac{1}{24 \varepsilon^{3}}\left[\left(m_{1}^{2}+m_{2}^{2}\right)^{2}+\left(m_{2}^{2}+m_{5}^{2}\right)^{2}+\left(m_{3}^{2}+m_{4}^{2}\right)^{2}\right. \\
& \left.+\left(m_{3}^{2}+m_{5}^{2}\right)^{2}-4\left(m_{2}^{2}-m_{3}^{2}\right)^{2}+m_{2}^{4}+m_{3}^{4}\right] \\
& +\mathcal{O}\left(\varepsilon^{-2}\right) .
\end{aligned}
$$

\section{Appendix B: Numerical input values}

$$
\begin{aligned}
m_{W} & =80.358 \mathrm{GeV}, \\
m_{u} & =2.2 \times 10^{-3} \mathrm{GeV}, \\
m_{d} & =4.8 \times 10^{-3} \mathrm{GeV}, \\
m_{c} & =1.4464 \mathrm{GeV}, \\
m_{s} & =0.093 \mathrm{GeV}, \\
m_{t} & =172.5 \mathrm{GeV}, \\
m_{b} & =4.8564 \mathrm{GeV}, \\
e & =0.30812, \\
\sin \theta_{w} & =0.47206 .
\end{aligned}
$$

\section{References}

1. G. Aad et al. (ATLAS Collaboration), Observation of a new particle in the search for the Standard Model Higgs boson with the ATLAS detector at the LHC. Phys. Lett. B 716, 1 (2012). https://doi.org/ 10.1016/j.physletb.2012.08.020

2. S. Chatrchyan et al. (CMS Collaboration), Observation of a new boson at a mass of $125 \mathrm{GeV}$ with the CMS experiment at the LHC. Phys. Lett. B 716, 30 (2012). https://doi.org/10.1016/j.physletb. 2012.08.021. arXiv:1207.7235 [hep-ex]

3. J.F. Gunion, H.E. Haber, G. Kane, S. Dawson, The Higgs Hunter's Guide (Westview Press, Boulder, 2000)

4. G.C. Branco, P.M. Ferreira, L. Lavoura, M.N. Rebelo, M. Sher, J.P. Silva, Theory and phenomenology of two-Higgs-doublet models. Phys. Rep. 516, 1 (2012). arXiv:1106.0034 [hep-ph]

5. I.P. Ivanov, Building and testing models with extended Higgs sectors. Prog. Part. Nucl. Phys. 95, 160-208 (2017). https://doi.org/ 10.1016/j.ppnp.2017.03.001. arXiv:1702.03776 [hep-ph]

6. P.A. Zyla et al. (Particle Data Group), Review of particle physics. PTEP 2020(8), 083C01 (2020). https://doi.org/10.1093/ ptep/ptaa104

7. L. Altenkamp, S. Dittmaier, H. Rzehak, Renormalization schemes for the two-Higgs-doublet model and applications to $h \rightarrow$ $W W / Z Z \rightarrow$ 4fermions. JHEP 09, 134 (2017). https://doi.org/ 10.1007/JHEP09(2017)134. arXiv:1704.02645 [hep-ph]

8. S.L. Glashow, S. Weinberg, Natural conservation laws for neutral currents. Phys. Rev. D 15, 1958 (1977). https://doi.org/10.1103/ PhysRevD.15.1958

9. E.A. Paschos, Diagonal neutral currents. Phys. Rev. D 15, 1966 (1977). https://doi.org/10.1103/PhysRevD.15.1966

10. G. Aad et al. (ATLAS and CMS Collaborations), Measurements of the Higgs boson production and decay rates and constraints on its couplings from a combined ATLAS and CMS analysis of the LHC pp collision data at $\sqrt{s}=7$ and 8 TeV. JHEP 1608, 045 (2016). https://doi.org/10.1007/ JHEP08(2016)045. arXiv:1606.02266 [hep-ex]

11. J.F. Gunion, H.E. Haber, The CP conserving two Higgs doublet model: the approach to the decoupling limit. Phys. Rev. D 67, 075019 (2003). https://doi.org/10.1103/PhysRevD.67.075019. arXiv:hep-ph/0207010

12. N. Cabibbo, Unitary symmetry and leptonic decays. Phys. Rev. Lett. 10, 531-533 (1963). https://doi.org/10.1103/PhysRevLett.10. 531

13. M. Kobayashi, T. Maskawa, CP violation in the renormalizable theory of weak interaction. Prog. Theor. Phys. 49, 652-657 (1973). https://doi.org/10.1143/PTP.49.652

14. I.F. Ginzburg, M. Krawczyk, Symmetries of two Higgs doublet model and CP violation. Phys. Rev. D 72, 115013115013 (2005). arXiv:hep-ph/0408011

15. A.W. El Kaffas, W. Khater, O.M. Ogreid, P. Osland, Consistency of the two Higgs doublet model and CP violation in top production at the LHC. Nucl. Phys. B 775, 45 (2007). arXiv:hep-ph/0605142

16. A. Arhrib, E. Christova, H. Eberl, E. Ginina, CP violation in charged Higgs production and decays in the Complex Two Higgs Doublet Model. JHEP 1104, 089 (2011). arXiv:1011.6560 [hep$\mathrm{ph}]$

17. A. Barroso, P.M. Ferreira, R. Santos, J.P. Silva, Probing the scalarpseudoscalar mixing in the $125 \mathrm{GeV}$ Higgs particle with current data. Phys. Rev. D 86, 015022 (2012). arXiv:1205.4247 [hep-ph]

18. S. Inoue, M.J. Ramsey-Musolf, Y. Zhang, CP violating phenomenology of flavor conserving two Higgs doublet models. Phys. Rev. D 89(11), 115023 (2014). arXiv:1403.4257 [hep-ph]

19. D. Fontes, J.C. Romão, J.P. Silva, $h \rightarrow Z \gamma$ in the complex two Higgs doublet model. JHEP 1412, 043 (2014). arXiv:1408.2534 [hep-ph]

20. B. Grzadkowski, O.M. Ogreid, P. Osland, Measuring CP violation in two-Higgs-doublet models in light of the LHC Higgs data. JHEP 1411, 084 (2014). arXiv:1409.7265 [hep-ph]

21. D. Fontes, M. Mühlleitner, J.C. Romão, R. Santos, J.P. Silva, J. Wittbrodt, The C2HDM revisited. JHEP 1802, 073 (2018). arXiv:1711.09419 [hep-ph]

22. R. Boto, T.V. Fernandes, H.E. Haber, J.C. Romão, J.P. Silva, Basis-independent treatment of the complex 2HDM. Phys. Rev. D 101(5), 055023 (2020). https://doi.org/10.1103/PhysRevD.101. 055023. arXiv:2001.01430 [hep-ph]

23. K. Cheung, A. Jueid, Y.N. Mao, S. Moretti, Phys. Rev. D 102(7), 075029 (2020). https://doi.org/10.1103/PhysRevD.102. 075029. arXiv:2003.04178 [hep-ph]

24. P.M. Ferreira, R. Santos, M. Sher, J.P. Silva, Could the LHC two-photon signal correspond to the heavier scalar in two-Higgsdoublet models? Phys. Rev. D 85, 035020 (2012). https://doi.org/ 10.1103/PhysRevD.85.035020. arXiv:1201.0019 [hep-ph]

25. J. Bernon, J.F. Gunion, H.E. Haber, Y. Jiang, S. Kraml, Scrutinizing the alignment limit in two-Higgs-doublet models. II. $\mathrm{m}_{H}=125$ GeV. Phys. Rev. D 93(3), 035027 (2016). https://doi.org/10.1103/ PhysRevD.93.035027. arXiv:1511.03682 [hep-ph]

26. F.J. Botella, J.P. Silva, Jarlskog-like invariants for theories with scalars and fermions. Phys. Rev. D 51, 3870-3875 (1995). https:// doi.org/10.1103/PhysRevD.51.3870. arXiv:hep-ph/9411288

27. H. Georgi, D.V. Nanopoulos, Suppression of flavor changing effects from neutral spinless meson exchange in gauge theories. Phys. Lett. B 82, 95-96 (1979). https://doi.org/10.1016/ 0370-2693(79)90433-7

28. J.F. Donoghue, L.F. Li, Properties of charged Higgs bosons. Phys. Rev. D 19, 945 (1979). https://doi.org/10.1103/PhysRevD.19.945

29. C. Jarlskog, Commutator of the quark mass matrices in the standard electroweak model and a measure of maximal CP violation. Phys. Rev. Lett. 55, 1039 (1985). https://doi.org/10.1103/PhysRevLett. 55.1039 
30. C. Jarlskog, A basis independent formulation of the connection between quark mass matrices, $\mathrm{CP}$ violation and experiment. $\mathrm{Z}$. Phys. C 29, 491-497 (1985). https://doi.org/10.1007/BF01565198

31. I. Dunietz, O.W. Greenberg, D.D. Wu, A priori definition of maximal CP violation. Phys. Rev. Lett. 55, 2935 (1985). https://doi.org/ 10.1103/PhysRevLett.55.2935

32. G.C. Branco, L. Lavoura, J.P. Silva, C.P. Violation, Int. Ser. Monogr. Phys. 103, 1-536 (1999)

33. M.E. Pospelov, I.B. Khriplovich, Electric dipole moment of the W boson and the electron in the Kobayashi-Maskawa model. Sov. J. Nucl. Phys. 53, 638-640 (1991)

34. M.J. Booth, The electric dipole moment of the $\mathrm{W}$ and electron in the Standard Model. arXiv:hep-ph/9301293

35. A. Pilaftsis, CP odd tadpole renormalization of Higgs scalarpseudoscalar mixing. Phys. Rev. D 58, 096010 (1998). https://doi. org/10.1103/PhysRevD.58.096010. arXiv:hep-ph/9803297

36. A. Denner, S. Dittmaier, Phys. Rep. 864, 1-163 (2020). https://doi. org/10.1016/j.physrep.2020.04.001. arXiv:1912.06823 [hep-ph]

37. H. Bélusca-Maĩto, A. Ilakovac, M. Mador-Božinović, D. Stöckinger, Dimensional regularization and BreitenlohnerMaison/'t Hooft-Veltman scheme for $\gamma_{5}$ applied to chiral YM theories: full one-loop counterterm and RGE structure. JHEP 08(08), 024 (2020). https://doi.org/10.1007/JHEP08(2020)024. arXiv:2004.14398 [hep-ph]

38. D. Fontes, J.C. Romão, FeynMaster: a plethora of Feynman tools. Comput. Phys. Commun. 256, 107311 (2020). https://doi.org/10. 1016/j.cpc.2020.107311. arXiv:1909.05876 [hep-ph]

39. N.D. Christensen, C. Duhr, FeynRules-Feynman rules made easy. Comput. Phys. Commun. 180, 1614-1641 (2009). https://doi.org/ 10.1016/j.cpc.2009.02.018. arXiv:0806.4194 [hep-ph]

40. P. Nogueira, Automatic Feynman graph generation. J. Comput. Phys. 105, 279-289 (1993). https://doi.org/10.1006/jcph.1993. 1074

41. T. Hahn, Generating Feynman diagrams and amplitudes with FeynArts 3. Comput. Phys. Commun. 140, 418-431 (2001). https:// doi.org/10.1016/S0010-4655(01)00290-9. arXiv:hep-ph/0012260

42. R. Mertig, M. Bohm, A. Denner, FEYN CALC: computer algebraic calculation of Feynman amplitudes. Comput. Phys. Commun. 64, 345-359 (1991). https://doi.org/10.1016/0010-4655(91)90130-D

43. V. Shtabovenko, R. Mertig, F. Orellana, New developments in FeynCalc 9.0. Comput. Phys. Commun. 207, 432-444 (2016). https://doi.org/10.1016/j.cpc.2016.06.008. arXiv:1601.01167 [hep-ph]

44. V. Shtabovenko, R. Mertig, F. Orellana, FeynCalc 9.3: new features and improvements. Comput. Phys. Commun. 256, 107478 (2020). https://doi.org/10.1016/j.cpc.2020.107478. arXiv:2001.04407 [hep-ph]
45. A.V. Smirnov, FIESTA4: optimized Feynman integral calculations with GPU support. Comput. Phys. Commun. 204, 189-199 (2016). https://doi.org/10.1016/j.cpc.2016.03.013. arXiv:1511.03614 [hep-ph]

46. A.V. Smirnov, F.S. Chuharev, FIRE6: Feynman Integral REduction with modular arithmetic. Comput. Phys. Commun. 247, 106877 (2020). https://doi.org/10.1016/j.cpc.2019.106877

47. S. Kanemura, T. Kubota, E. Takasugi, Lee-Quigg-Thacker bounds for Higgs boson masses in a two doublet model. Phys. Lett. B 313, 155-160 (1993). https://doi.org/10.1016/0370-2693(93)91205-2. arXiv:hep-ph/9303263

48. A.G. Akeroyd, A. Arhrib, E.M. Naimi, Note on tree level unitarity in the general two Higgs doublet model. Phys. Lett. B 490, 119124 (2000). https://doi.org/10.1016/S0370-2693(00)00962-X. arXiv:hep-ph/0006035

49. I.F. Ginzburg, I.P. Ivanov, Tree-level unitarity constraints in the most general 2HDM. Phys. Rev. D 72, 115010 (2005). https://doi. org/10.1103/PhysRevD.72.115010. arXiv:hep-ph/0508020

50. P. Bechtle, O. Brein, S. Heinemeyer, O. Stål, T. Stefaniak, G. Weiglein, K.E. Williams, HiggsBounds - 4: improved tests of extended Higgs sectors against exclusion bounds from LEP, the Tevatron and the LHC. Eur. Phys. J. C 74(3), 2693 (2014). https:// doi.org/10.1140/epjc/s10052-013-2693-2. arXiv:1311.0055 [hep$\mathrm{ph}]$

51. P. Bechtle, D. Dercks, S. Heinemeyer, T. Klingl, T. Stefaniak, G. Weiglein, J. Wittbrodt, HiggsBounds-5: testing Higgs sectors in the LHC $13 \mathrm{TeV}$ era. Eur. Phys. J. C 80(12), 1211 (2020). https:// doi.org/10.1140/epjc/s10052-020-08557-9

52. G. Aad et al. (ATLAS), Combined measurements of Higgs boson production and decay using up to $80 \mathrm{fb}^{-1}$ of proton-proton collision data at $\sqrt{s}=13 \mathrm{TeV}$ collected with the ATLAS experiment. Phys. Rev. D 101(1), 012002 (2020). https://doi.org/10.1103/ PhysRevD.101.012002. arXiv:1909.02845 [hep-ex]

53. S. Bauberger, A. Freitas, TVID: three-loop vacuum integrals from dispersion relations. arXiv:1702.02996 [hep-ph]

54. S. Bauberger, A. Freitas, D. Wiegand, TVID 2: evaluation of planar-type three-loop self-energy integrals with arbitrary masses. JHEP 01, 024 (2020). https://doi.org/10.1007/JHEP01(2020)024. arXiv:1908.09887 [hep-ph]

55. S.P. Martin, D.G. Robertson, Evaluation of the general 3-loop vacuum Feynman integral. Phys. Rev. D 95(1), 016008 (2017). https://doi.org/10.1103/PhysRevD.95.016008. arXiv:1610.07720 [hep-ph]

56. A. Freitas, Three-loop vacuum integrals with arbitrary masses. JHEP 11, 145 (2016). https://doi.org/10.1007/JHEP11(2016)145. arXiv:1609.09159 [hep-ph] 